\title{
Nanopatterned Self-Assembled Monolayers by Using Diblock Copolymer Micelles as Nanometer-Scale Adsorption and Etch Masks
}

\author{
By Sivashankar Krishnamoorthy, Raphael Pugin, Juergen Brugger, Harry Heinzelmann, \\ and Christian Hinderling*
}

Nanometer-scale patterning of surfaces is a highly relevant and interesting topic of research today, owing to its potential contributions to device miniaturization and creation of novel functional interfaces with applications ranging from electronics to biomedicine. Several methods of nanopatterning are known, for example, nanoimprint lithography, ${ }^{[1]}$ laser interferometry, ${ }^{[2]}$ extreme ultraviolet interference lithography, ${ }^{[3,4]}$ shadow mask lithography, ${ }^{[5,6]}$ and scanning-probe-microscopy-based lithographic methods. ${ }^{[7-9]}$ Although the topographical and chemical structuring of surfaces by using one or more of these techniques is well-established and is state-of-the-art, these methods also suffer from some drawbacks. They either involve a costly mask fabrication step, heavy infrastructure investments, clean-room conditions, or are restricted to the patterning of comparatively small areas only. One alternative method of surface structuring that addresses some of these drawbacks is block copolymer lithography, where use is made of polymer masks derived from selfassembly of block copolymers. ${ }^{[10-12]}$ This process has gained popularity owing to facile fabrication of sub-100 nm structures on macroscopic areas. The preparation of nanometer-scale masks by using phase-separated block copolymer thin films frequently involves working with appropriate film thickness and surface energy, an annealing step to order the polymer domains, and selective degradation and removal of one of the domains. ${ }^{[13]}$ Many of the advantages of this method can also be realized in a much simplified approach by using block copolymer micelles deposited from solution. ${ }^{[14]}$ The attractiveness of the latter approach lies in the fact that the micellar films can be readily prepared on a wide variety of substrates, and in addition, their dimensions and spacing can be tuned in a straight-forward manner, without having to change the polymer molecular weights. ${ }^{[15,16]}$ Micellar thin films, even in their as-coated form, can be used for structuring surfaces

[*] Dr. C. Hinderling, Dr. S. Krishnamoorthy, Dr. R. Pugin, Dr. H. Heinzelmann

Nanotechnology \& Life Sciences section

Swiss Center for Electronics and Microtechnology SA (CSEM SA)

Neuchatel 2000 (Switzerland)

E-mail: chi@csem.ch

Prof. J. Brugger

Microsystems Laboratory (LMIS)

Ecole Polytechnique Federale de Lausanne

Ecublens 1015 (Switzerland) without the need for additional processing steps. We report here an interesting approach to create nanopatterned selfassembled monolayers (SAMs) with feature dimensions in the sub- $100 \mathrm{~nm}$ regime, and with periodicity of $100 \mathrm{~nm}$, starting from thin films of polystyrene-block-poly(2-vinylpyridine) (PS- $b$-P2VP) copolymer reverse micellar thin films on silicon surfaces.

Self-assembled monolayers have served as an attractive means of chemically modifying surfaces for various applications like controlling surface wettability, structuring surfaces, and binding of species such as metal ions, nanoparticles, and biomolecules. ${ }^{[17-19]}$ Formation of monolayers through covalent binding of silane molecules on silicon surfaces is a well-studied and mature concept that is particularly attractive for the mechanical and thermal stability of the silane monolayers in addition to prevalence in use of silicon for microelectromechanical systems (MEMS). Silanes and other monolayer-forming molecules have been patterned by using top-down techniques such as microcontact printing, ${ }^{[20,21]}$ photolithography, ${ }^{[22]}$ and nanoimprinting ${ }^{[23]}$ with interests for selective surface modification and achieving chemical and/or wettability contrast. Earlier work has shown the use of nanosphere lithography to create chemical nanopatterns on surfaces. ${ }^{[2-26]}$ Although interesting, this approach suffers from disadvantages arising from difficulty in achieving homogenous 2D colloidal monolayers over large areas of surface. Also, the creation of high-density nanopatterns with feature-to-feature separations on the order of $100 \mathrm{~nm}$ would require the use of particles with sizes of $100 \mathrm{~nm}$ or below, making it more difficult to obtain large-scale patterns. The copolymer micelle lithography approach that we present here provides a facile and highly reproducible means of achieving nanopatterned SAMs with excellent homogeneity and integrity over whole wafer areas. We demonstrate the use of such a nanopatterned SAM for the fabrication of surfaces that act as universal templates for the controlled creation of arrays of nanocrystals.

A monolayer of spherical reverse micelles of PS- $b$-P2VP can be obtained on a surface by spin-coating a dilute solution of the copolymer in solvents such as toluene and xylene that are selective for the PS block. The micelles self-organize on the surface to form quasi-hexagonal periodic arrays that exhibit a nanometer-scale contrast in topography. The topographic contrast offered by the micelle thin films in their as-coated form offers attractive opportunities to transfer the micellar 

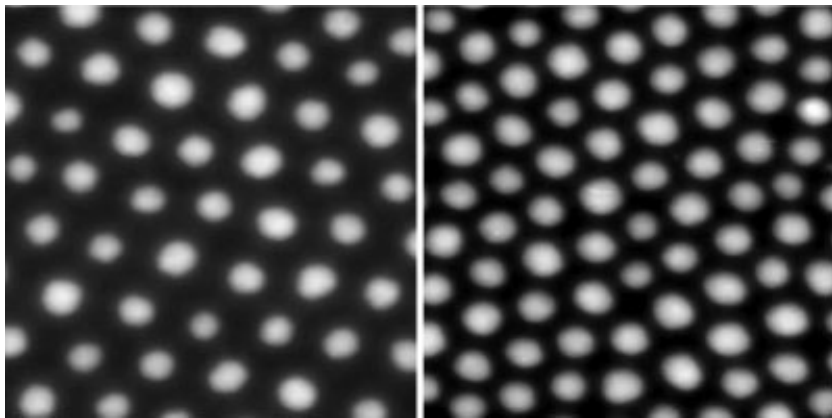

Figure 1. Tapping mode atomic force microscopy images $\left(1 \times 1 \mu \mathrm{m}^{2}\right.$ scan size) of an array of PS-b-P2VP reverse micelles spin-coated on silicon surfaces with different periodicities obtained by systematically varying the coating conditions.

pattern into the underlying substrate. We have shown earlier the use of copolymer micelle thin films as etch masks to structure silicon surfaces to create pillars and holes. ${ }^{[27]}$ In this Communication, we show that the topographic contrast provides convenient means of creating nanometer-scale polymer islands that subsequently act as "masks" preventing access to the underlying substrate for adsorbing or etching species. We demonstrate formation of nanopatterns of SAMs of silane molecules by exploiting micellar thin-film-derived polymer masks as either adsorption or etch masks. The periodicity of these masks mirror that of the as-coated micelle thin films, and hence are fine-tunable by varying the coating conditions such as spin-coating velocities and concentration of the polymer solution (Fig. 1). ${ }^{[16]}$

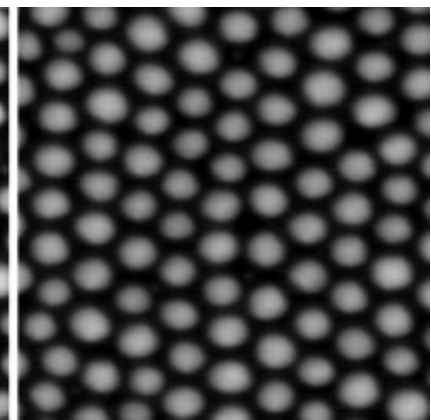

In our first approach for fabricating nanopatterned SAMs, micelles are used as adsorption masks. A monolayer of reverse micelles of PS- $b$-P2VP (91500$b-105000 \mathrm{~g} \mathrm{~mol}^{-1}$ ) in $o$-xylene solution is deposited on a freshly piranha treated silicon surface by spin-coating at $5000 \mathrm{rpm}$. This results in the formation of thin films of micelles that reveals an array of bumps with heights of $20 \mathrm{~nm}$ (as measured by atomic force microscopy (AFM)), with a periodicity of $100 \mathrm{~nm}$, and with a continuous intermediate polymer layer of $10 \mathrm{~nm}$ thickness in between the bumps that formed from fusion of the micelle coronas. The micellar thin film is then subjected to a brief oxygen plasma step $\left(30 \mathrm{~W}, 50 \mathrm{~m}\right.$ Torr $\left(1\right.$ Torr $\left.\left.=1.333 \times 10^{2} \mathrm{~Pa}\right), 30 \mathrm{~s}\right)$ to remove the intermediate layer between the bumps and expose the silicon beneath. The power and duration of the plasma exposure was chosen such that it resulted in removal of approximately $10 \mathrm{~nm}$ of the polymer only. This is sufficient to remove the continuous thin intermediate layer of polymer and formation of nanometer-scale polymer masks with an average height of $10 \mathrm{~nm}$ and periodicity same as that of the original micelle array. The subsequent exposure of this surface to $n$-octyldimethylchlorosilane vapors results in selective silanization on the silicon exposed regions and masking of areas

Micelles as adsorption masks

a)

PS-b-P2VP Micelle
monolayer

After brief oxygen plasma

Silane adsorption (using noctyldimethylchlorosila ne vapors)

Patterned SAM

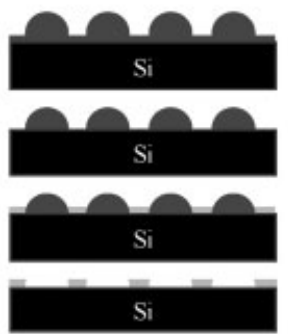

b)

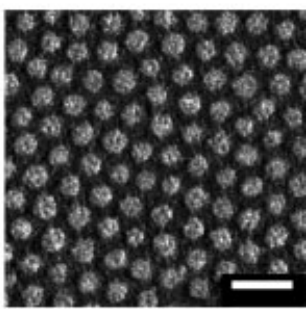

\section{Micelles as etch masks}

c)

$$
\begin{aligned}
& \text { PS-b-P2VP micellar monolayer } \\
& \text { on top of OTS SAM }
\end{aligned}
$$

Structure transfer into underlying SAM

nanopatterned SAM

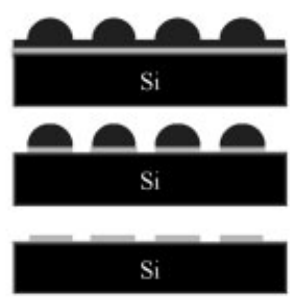

d)

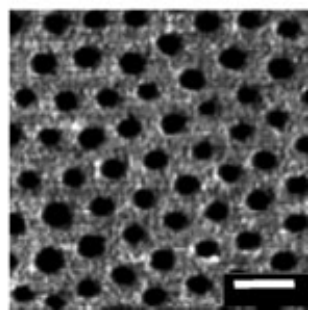

Figure 2. a,c) Schematic illustration of the process steps involved in creating structured SAMs using two different approaches. b,d) Friction-mode AFM measurements on the structured SAMs. The darker regions in the friction mode image correspond to lower-friction areas consisting of hydrophobic silane SAM. Scale bar indicates $200 \mathrm{~nm}$. 
under the polymer masks (Fig. 2a). The polymer masks were lifted off by washing with dimethyl sulfoxide (DMSO) followed by isopropyl alcohol to obtain the nanopatterned SAMs (Fig. 2b). The complete removal of the polymer masks was confirmed using atomic force microscopy measurements in friction and phase mode, which revealed a distinct nanoscale pattern with $60 \mathrm{~nm}$ feature dimensions (Fig. 2b). The AFM tip experiences less friction on the hydrophobic SAM-covered areas and higher friction on silicon-exposed areas. This transforms into silane-covered regions (matrix) appearing dark and silicon-exposed regions (circular patches) appearing bright in the friction mode images, which is in accordance with the expected contrast for the patterns prepared.

In the second approach employed for creating nanopatterned SAMs, the micelle thin films were used as etch masks to structure an underlying SAM. A monolayer of PS- $b$-P2VP micelles was formed on octadecyltrichlorosilane (OTS) pretreated silicon surface followed by exposure to brief oxygen plasma $(30 \mathrm{~W}, 50 \mathrm{mTorr}, 40 \mathrm{~s})$. The oxygen plasma exposure was slightly longer than for the previous case, and was sufficient to transfer the micelle nanopattern into the underlying OTS SAM. The remaining polymer masks were lifted off using DMF and isopropyl alcohol resulting in ordered circular islands of OTS SAM with an average diameter of ca. $50 \mathrm{~nm}$ on silicon surface (Fig. 2c). The characterization of the nanopatterned SAMs was carried out with AFM measurements in the friction (Fig. 2d) and phase modes (not shown). It can be seen that this approach yields nanopatterns complementary to those achieved using the earlier approach. The silane coveredregions (now the circular patches) appear dark and silicon-exposed regions (matrix) appear bright in the friction-mode images.

We further demonstrate the use of wettability contrast offered by the nanopatterned SAMs for fast and flexible creation of arrays of nanoparticles of controlled particle size by selective dewetting of aqueous salt solutions within the nanopatterns. In a typical experiment, a silicon substrate with a nanopatterned SAM consisting of hydrophilic Si-patches in a hydrophobic silane matrix (obtained using the first approach sketched above) into a solution of $1 \mathrm{~m} \mathrm{NaCl}$ aqueous solution. Upon removal of the substrate from the solution, the surface emerges dry owing to solution de-wetting. The surface was blown briefly with $\mathrm{N}_{2}$ gas to ensure removal of any remaining

a)

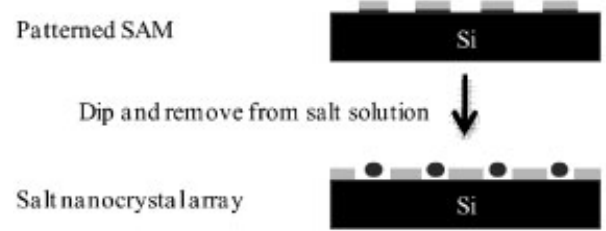

b)

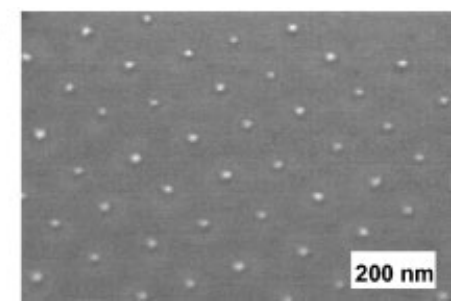

Figure 3. Wettability contrast offered by a continuous matrix of hydrophobic SAM with the hydrophilic silicon surface exposed within ordered circular regions is used for selective crystallization of sodium chloride crystals by simple dipping and removal of the substrate from aqueous salt solution. a) Schematic of the process, and b) SEM of a $\mathrm{NaCl}$ nanocrystal array. moisture. The analysis of the surface by SEM reveals the presence of nanoparticles in regular arrays, as shown in Figure 3. The formation of the particles was observed over the entire patterned area with excellent homogeneity. The heights of the particles were determined to be $3.5 \mathrm{~nm}$ by using AFM topography measurements.

In conclusion, we describe what we believe to be a very general method of creating high-density surface energy nanopatterns. The two approaches described above lead to complementary nanopatterns, thus making the procedures even more flexible. Together with the already communicated ability of continuously tuning the micelle spacing this approach forms a very general tool. We further report on first results of a particular application of such surfaces to template the creation of nanoparticle arrays. We believe that this method of creating particle arrays to be of very wide scope. It unifies several attractive features such as the ability to control the average distance of the particles by varying micelle array periodicity, the ability to control the size of the particle by varying the solution concentration, and also the ability to control the chemistry of the particle by the solution composition. Taking this thought a bit further it might even be possible to create more than one particle per spot by dipping it into different solutions in sequence. Depending on the nature of the particles, interesting interparticle phenomena could result. A further potentially important application of such patterned surfaces lies in the control of crystallization processes.

\section{Experimental}

n-Octyl dimethylchlorosilane and octadecyltrichlorosilane (OTS) were obtained from ABCR (Karlsruhe, Germany). Polystyrene-blockpoly(2-vinylpyridine) with molecular weight of $91500-b-105000 \mathrm{~g} \mathrm{~mol}^{-1}$ and a polydispersity indey (PDI) of 1.1 was obtained from Polymer Source Inc. (Montreal, Canada) and was used as-received. The silicon substrates used for the experiments were cleaned in boiling piranha solution at $130{ }^{\circ} \mathrm{C}$ for 10 mins and subsequently washed thoroughly with millipore water. The piranha solution was prepared by mixing 1:1 volume ratio of $\mathrm{H}_{2} \mathrm{O}_{2}$ and con. $\mathrm{H}_{2} \mathrm{SO}_{4}$ (Warning: Piranha solutions should be handled carefully during preparation and disposal because of explosion hazards).The micelle solution preparation and coating conditions were followed as reported in Ref. ${ }^{[14]}$. The coating of the micelles was carried out at a atmospheric humidity of $15-20 \%$. Silanization was carried out through exposure of the surface to vapors of silane, in a vacuum desiccator, for duration of $2 \mathrm{~h}$ for OTS SAMs and $3.5 \mathrm{~h}$ for $n$-octyldimethylchlorosilane SAMs, followed by thorough washing with hexane and isopropyl alcohol. The removal of the polymer masks was best achieved by mild swabbing of the surface placed under DMSO with a cotton swab, followed by thorough washing with isopropyl alcohol. The quality of nanopatterns was found unaffected by such a treatment. The AFM measurements were performed with Veeco Dimension 3100 instrument and controlled by Nanoscope software. An Oxford Plasmalab80plus machine (Oxford Instruments, Bristol, UK) was used 
for the oxygen plasma treatment. Friction-force measurements were carried out using V-shaped DNP-S type silicon nitride cantilevers from Veeco Instruments Inc. (CA, USA). Scanning electron microscopy was performed using Philips XL-30 ESEM-FEG instrument.

Received: August 10, 2007 Revised: December 23, 2007 Published online:

[1] L. J. Guo, Adv. Mater. 2007, 19, 495.

[2] C. J. M. van Rijn, J. Microlithogr. Microfabr. Microsyst. 2006, 5, 011012.

[3] H. H. Solak, J. Phys. D 2006, 39, R171.

[4] J. I. Martin, J. Nogues, K. Liu, J. L. Vicent, I. K. Schuller, J. Magn. Magn. Mater. 2003, 256, 449.

[5] R. Luthi, R. R. Schlittler, J. Brugger, P. Vettiger, M. E. Welland, J. K. Gimzewski, Appl. Phys. Lett. 1999, 75, 1314.

[6] J. Brugger, J. W. Berenschot, S. Kuiper, W. Nijdam, B. Otter, M. Elwenspoek, Microelectron. Eng. 2000, 53, 403.

[7] G. Y. Liu, S. Xu, Y. L. Qian, Acc. Chem. Res. 2000, 33, 457.

[8] S. Kramer, R. R. Fuierer, C. B. Gorman, Chem. Rev. 2003, 103, 4367.

[9] R. D. Piner, J. Zhu, F. Xu, S. H. Hong, C. A. Mirkin, Science 1999, 283, 661.

[10] C. Harrison, M. Park, P. M. Chaikin, R. A. Register, D. H. Adamson, J. Vac. Sci. Technol. B 1998, 16, 544.
[11] P. Mansky, C. K. Harrison, P. M. Chaikin, R. A. Register, N. Yao, Appl. Phys. Lett. 1996, 68, 2586.

[12] M. Park, C. Harrison, P. M. Chaikin, R. A. Register, D. H. Adamson, Science 1997, 276, 1401.

[13] I. W. Hamley, Nanotechnology 2003, 14, R39.

[14] J. C. Meiners, A. Ritzi, M. H. Rafailovich, J. Sokolov, J. Mlynek, G. Krausch, Appl. Phys. A 1995, 61, 519.

[15] Y. S. Yu, A. Eisenberg, J. Am. Chem. Soc. 1997, 119, 8383.

[16] S. Krishnamoorthy, R. Pugin, M. Liley, H. Heinzelmann, J. Brugger, C. Hinderling, Adv. Funct. Mater. 2006, 16, 1469.

[17] A. Ulman, Chem. Rev. 1996, 96, 1533.

[18] J. C. Love, L. A. Estroff, J. K. Kriebel, R. G. Nuzzo, G. M. Whitesides, Chem. Rev. 2005, 105, 1103

[19] S. F. Chen, L. Y. Liu, J. Zhou, S. Y. Jiang, Langmuir 2003, 19, 2859.

[20] N. L. Jeon, R. G. Nuzzo, Y. Xia, M. Mrksich, G. M. Whitesides, Langmuir 1995, 11, 3024.

[21] J. Aizenberg, A. J. Black, G. M. Whitesides, Nature 1999, 398, 495.

[22] J. F. Mooney, A. J. Hunt, J. R. McIntosh, C. A. Liberko, D. M. Walba, C. T. Rogers, Proc. Natl. Acad. Sci.USA 1996, 93, 12291.

[23] L. Ressier, C. Martin, B. Viallet, J. Grisolia, J. P. Peyrade, J. Vac. Sci. Technol. B 2007, 25, 17.

[24] H. Agheli, J. Malmstrom, E. M. Larsson, M. Textor, D. S. Sutherland, Nano Lett. 2006, 6, 1165.

[25] A. Valsesia, P. Colpo, T. Meziani, P. Lisboa, M. Lejeune, F. Rossi, Langmuir 2006, 22, 1763

[26] Y. Cai, B. M. Ocko, Langmuir 2005, 21, 9274

[27] S. Krishnamoorthy, R. Pugin, J. Brugger, H. Heinzelmann, A. C. Hoogerwerf, C. Hinderling, Langmuir 2006, 22, 3450. 